\title{
The utility of ultrasound in the assessment of traumatic peripheral nerve lesions: report of 4 cases
}

\author{
Joshua Zeidenberg, MD, ${ }^{1}$ S. Shelby Burks, MD, ${ }^{2}$ Jean Jose, DO,' Ty K. Subhawong, MD,1 and \\ Allan D. Levi, MD, PhD² \\ 1Department of Radiology, University of Miami/Jackson Memorial Hospital; and 2Department of Neurological Surgery and The \\ Miami Project to Cure Paralysis, University of Miami Miller School of Medicine, Miami, Florida
}

\begin{abstract}
Ultrasound technology continues to improve with better image resolution and availability. Its use in evaluating peripheral nerve lesions is increasing. The current review focuses on the utility of ultrasound in traumatic injuries. In this report, the authors present 4 illustrative cases in which high-resolution ultrasound dramatically enhanced the anatomical understanding and surgical planning of traumatic peripheral nerve lesions. Cases include a lacerating injury of the sciatic nerve at the popliteal fossa, a femoral nerve injury from a pseudoaneurysm, an ulnar nerve neuroma after attempted repair with a conduit, and, finally, a spinal accessory nerve injury after biopsy of a supraclavicular fossa lesion. Preoperative ultrasound images and intraoperative pictures are presented with a focus on how ultrasound aided with surgical decision making. These cases are set into context with a review of the literature on peripheral nerve ultrasound and a comparison between ultrasound and MRI modalities.
\end{abstract}

http://thejns.org/doi/abs/10.3171/2015.6.FOCUS15214

KEY WORDS ultrasound; peripheral nerves; tibial nerve; peroneal nerve; ulnar nerve; spinal accessory nerve

$\mathrm{P}$ ERIPHERAL nerve injuries can be classified according to their location, mechanism of injury, and completeness of injury. Neurological impairment to the extremities includes motor and sensory loss as well as the potential for the development of neuropathic pain. ${ }^{1}$ In the setting of trauma, peripheral nerve lesions often represent one of the most serious long-term sequelae affecting quality of life and causing disability. ${ }^{21}$ In a study of 5777 patients with trauma, Noble et al. ${ }^{20}$ found a $3 \%$ prevalence of traumatic peripheral nerve injuries, 54\% of which required surgical intervention. Taylor et al. ${ }^{23}$ found a $1.64 \%$ incidence of traumatic peripheral nerve injury, with the highest rate of such pathology seen with crush injuries.

The diagnosis and localization of peripheral nerve lesions rely primarily on clinical history and physical/neurological examination. ${ }^{16}$ Electromyography (EMG) and nerve conduction studies are useful in confirming the completeness of peripheral nerve injury but cannot differentiate between axonotmesis and neurotmesis. ${ }^{24}$ Even the combination of neurological examination and EMG is insufficient for determining the precise extent of nerve damage and whether to proceed conservatively or with surgical repair. ${ }^{24}$ Although the absence of significant neurological recovery after a 4- to 6-month observation period is one of the critical determinants for surgical exploration after blunt nerve injury, obtaining preoperative anatomical information of the status of the nerve injury is becoming more important in surgical planning. Critical information to be gleaned from ultrasound includes the following: 1) whether the nerve remains in continuity; 2) length of the gap, if present; 3 ) presence of a focal neuroma and its location/size; 4) additional areas of nerve injury, such as tandem lesions; 5) degree of adjoining scar tissue; and 6) status of adjoining tissues such as blood vessels and bone.

Direct imaging with MRI and ultrasonography (US) is widely used when evaluating the extent of peripheral nerve injuries. MRI provides high-resolution imaging of peripheral nerves using $\mathrm{T} 2$-weighted images in combination with 
fat and flow suppression, which yield excellent representations of peripheral nerve anatomy. ${ }^{8,16} \mathrm{MRI}$, however, is limited in some centers by the difficulties of access, cost, and time. ${ }^{15}$ High-resolution ultrasonography (HRU) has been shown to be a useful tool in the diagnosis of peripheral nerve lesions. ${ }^{1,3,5,16,21,26} \mathrm{HRU}$ can easily identify all the main nerve trunks running in the limbs, including the median, ulnar, and radial nerves in the upper limbs and the sciatic, common peroneal, and posterior tibial nerves in the lower limbs. ${ }^{3}$ Review of the literature shows that ultrasound is successful in clearly and accurately demonstrating complete or partial transections, nerve lacerations, epineural hematoma, and neuroma formation as well as the adequacy of postsurgical repair., 9,10,16,21,22,24,26

In this paper, we present 4 cases in which HRU accurately demonstrated the anatomy and extent of traumatic peripheral nerve injury and guided the surgical management for these patients.

\section{Case Reports}

\section{Case 1}

A 16-year-old boy presented to our neurosurgery clinic 1 month after sustaining a lacerating injury to his left thigh at the superior border of the popliteal fossa. At the initial presentation, primary repair of the tibial nerve was attempted, and the leg was splinted at $45^{\circ}$ of flexion. In the clinic, he complained of severe pain in the distribution of

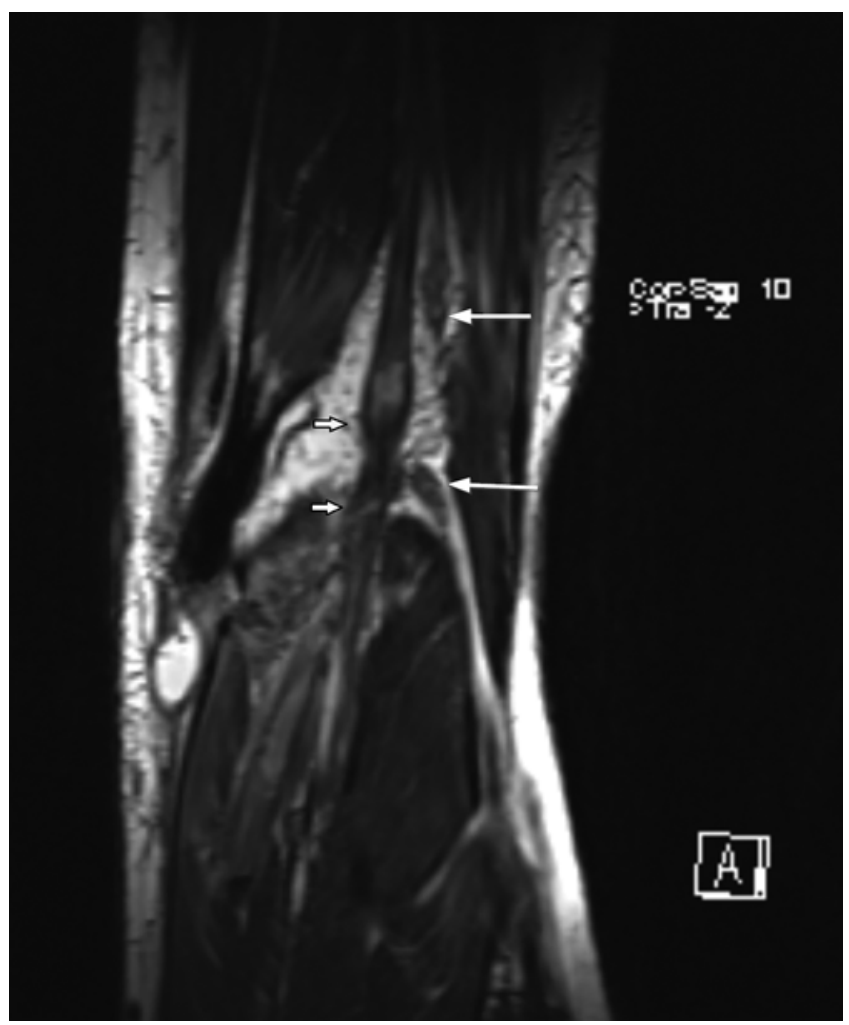

FIG. 1. Coronal T2 SPACE (sampling perfection with application optimized contrasts using different flip angle evolution) sequences through the level of the knee revealing laceration of the tibial and common peroneal nerves. There is a gap between the ends of the lacerated tibial nerve (short arrows) and lacerated common peroneal nerve (long arrows). the sciatic nerve, and physical examination showed Grade 0/5 strength of ankle dorsiflexion, eversion, plantar flexion, and extensor hallucis longus. There was absent sensation on the lateral leg and both surfaces of the foot. EMG studies revealed a lack of distal reinnervation in leg musculature for both tibial and peroneal distributions. MRI revealed evidence of a laceration of the tibial and common peroneal nerves resulting in complete transection (Fig. 1). Diagnostic ultrasound performed on the same date showed complete transection of these nerves at the level of the popliteal fossa and early formation of end-bulb neuromas (Fig. 2). The tibial graft had likely failed as a result of tension between the 2 nerve endings. These findings were confirmed intraoperatively (Fig. 3). The patient underwent neuroma resection and end-to-end grafting with 6 autologous sural nerve grafts applied to the tibial nerve, each spanning $4 \mathrm{~cm}$, and 4 similar grafts, $6 \mathrm{~cm}$ in length, were applied to the peroneal nerve.

\section{Case 2}

A 74-year-old man presented to the neurosurgery clinic 3 months after undergoing a percutaneous femoral artery approach for a cardiac ablation procedure. The patient reported experiencing immediate left proximal leg weakness after the procedure and numbness along the medial leg. On physical examination of the left lower extremity, he had Grade 3/5 strength on hip flexion and Grade 1/5 strength on knee extension. He had diminished sensation
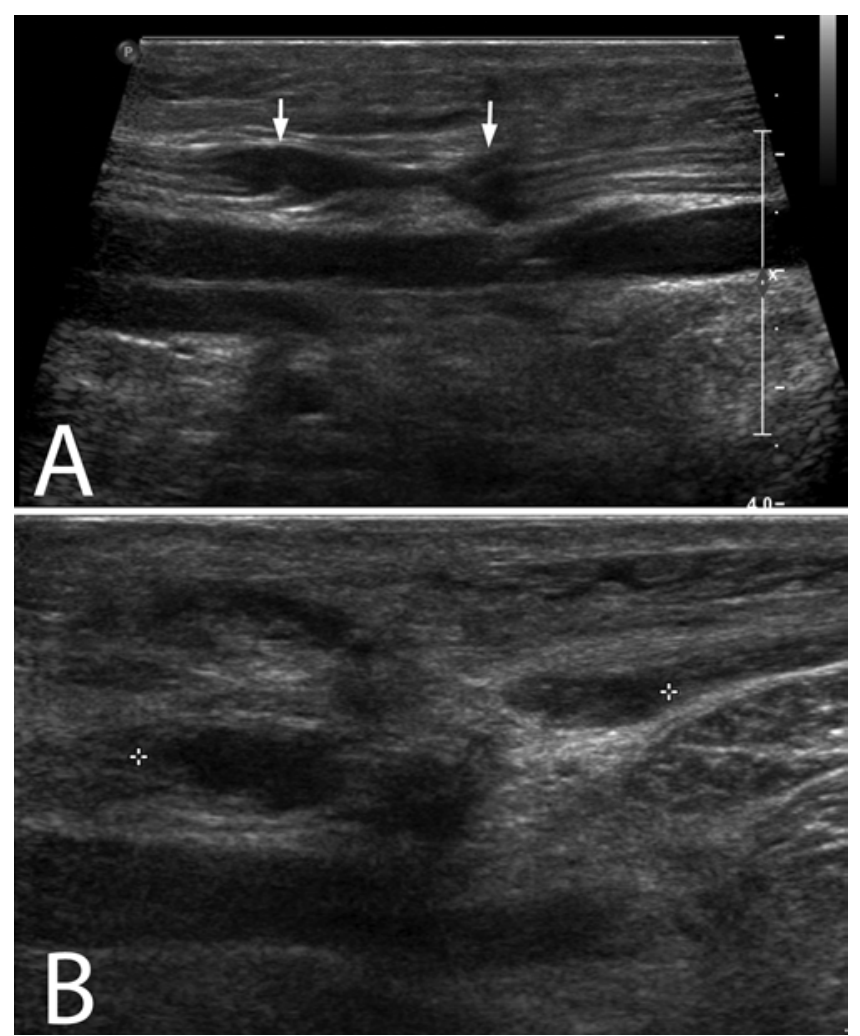

FIG. 2. A: HRU image of the tibial nerve reveals scar tissue at the level of transection (arrows) measuring $3 \mathrm{~cm}$, inclusive of end-bulb neuroma formation at the proximal and distal stump margins. B: Similarly, the gap between the transected ends of the common peroneal nerve is $3 \mathrm{~cm}$ (calipers). 


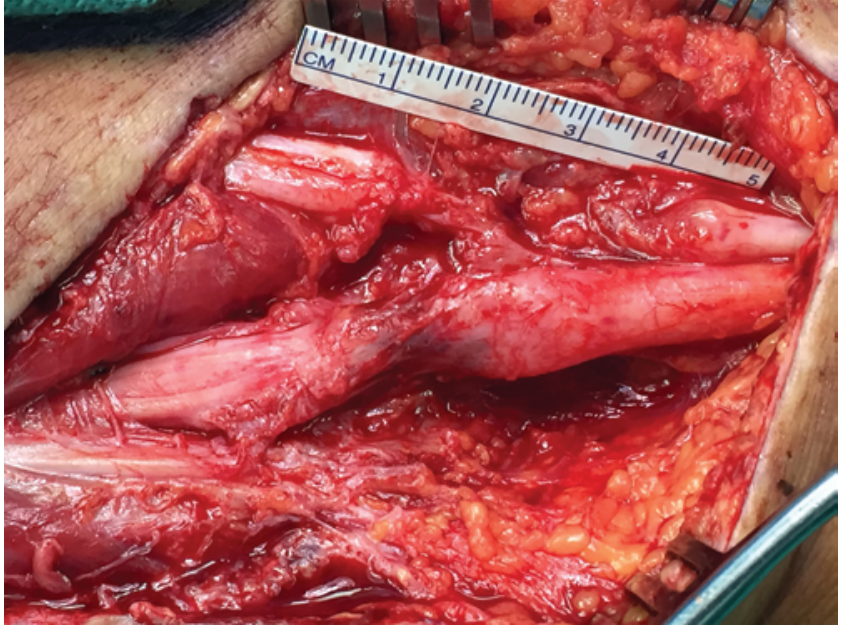

FIG. 3. Intraoperative image of tibial (inferior) and peroneal (superior) nerves at their bifurcation from the sciatic nerve and their course distally. The lateral head of the gastrocnemius splits the 2 nerves as they travel to the leg. The lacerating injury to both nerves is apparent in the significant gaps. In addition, enlargement of the distal stumps can be seen. This neuromatous enlargement appears most prominent in the tibial branch.

along the saphenous nerve distribution. MRI showed a heterogeneous mass arising off the left profunda femoral artery that appeared to abut and dissect into the adjacent femoral nerve, which itself showed signal abnormality (Fig. 4). Ultrasound performed on the same day showed a pseudoaneurysm dissecting and displacing the left femoral nerve (Fig. 5). The patient underwent surgery for vascular repair and neurolysis of the left femoral nerve with sural nerve grafting (Fig. 6). The patient showed clinical improvement of his symptoms with improved correlative EMG findings 3 months after surgery.

\section{Case 3}

A 20-year-old $\operatorname{man}^{4}$ sustained a complex wrist fracture/ dislocation that required open-reduction internal fixation. He presented to the neurosurgery clinic after this repair with left hand tingling and numbness in an ulnar nerve distribution and also profound grip weakness. Three months after the injury, he underwent surgery for exploration and repair with a $1.7-\mathrm{cm}$ decellularized allograft. At the 8-month follow-up, there was no clinical or electrophysiological evidence of reinnervation of the ulnar nerve graft. The patient complained of persistent tingling and numbness, grip weakness, and progressive clawing of the hand. He had marked atrophy of the intrinsic muscles of the hand and absent sensation in the ulnar distribution. The patient again underwent surgery for reexploration, at which time an end-bulb neuroma was resected and sural nerve grafts were placed (Fig. 7B). Postoperative ultrasound effectively showed continuity of the nerve graft with no evidence of neuroma formation 6 or 12 months after surgery (Fig. 7C). The patient improved clinically with concordant EMG findings. ${ }^{4}$

\section{Case 4}

The final presented case is of a 62-year-old woman who underwent a biopsy of a right-sided neck lymph node and

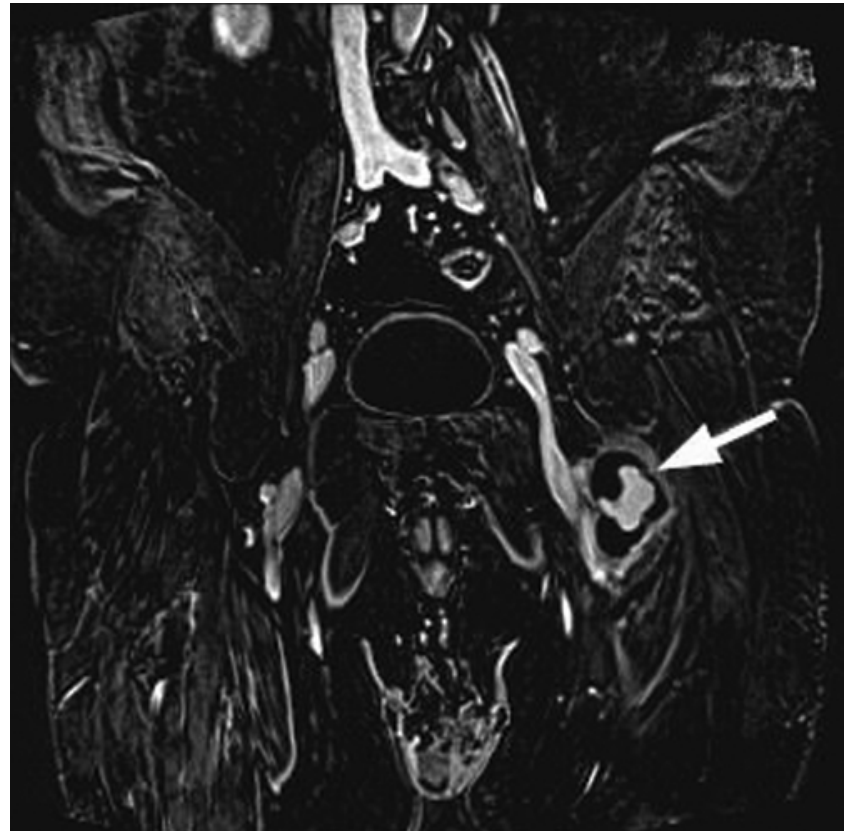

FIG. 4. Coronal T2-weighted image of the pelvis shows a heterogeneous signal intensity mass abutting and dissecting into the left femoral nerve (arrow). The central enhancement and peripheral signal void represent the to-and-fro blood flow of the iatrogenic pseudoaneurysm.

presented to the neurosurgery clinic with complaints of right neck, shoulder, and arm pain (score 9 of 10) that began the day after surgery. The pain was constant and worse with activity. She also complained of upper-arm weakness and limited range of motion. Her physical examination demonstrated Grade 3/5 weakness and atrophy of a portion of the right trapezius. EMG revealed 1+ fibrillations (e.g., see http://courses.kcumb.edu/physio/2008\%20EMG/ cmap.htm) and decreased innervation of the spinal accessory nerve on the right in the corresponding musculature, which was $35 \%$ of the response compared with that of the normal left side. Ultrasound of the right supraclavicular fossa was performed, which revealed a Sunderland Grade 5 lesion involving the spinal accessory nerve with a 1.5$\mathrm{cm}$ gap between the severed nerve ends and a 4-mm endbulb neuroma (Fig. 8). In light of the ultrasound findings and her residual neurological function, the mutual decision to proceed with surgical repair of the right spinal accessory nerve was made.

\section{Discussion}

Over half of all peripheral nerve injuries in the setting of trauma require surgical intervention..$^{20}$ The precise localization of the site and extent of injury may remain uncertain after physical examination and EMG testing. ${ }^{24}$ Ultrasound has been shown to be an efficient, noninvasive, and low-cost method of imaging traumatic peripheral nerve pathologies. ${ }^{9}, 10,16,21,22,24,26$ After trauma, HRU can differentiate between rupture of a nerve bundle and fibroblast infiltration, which results in the formation of a traumatic neuroma. ${ }^{24}$ Padua et al. ${ }^{21}$ showed that ultrasound was able to aid in the diagnosis or modification of the therapeutic path in $60 \%$ of patients with traumatic nerve lesions and, 


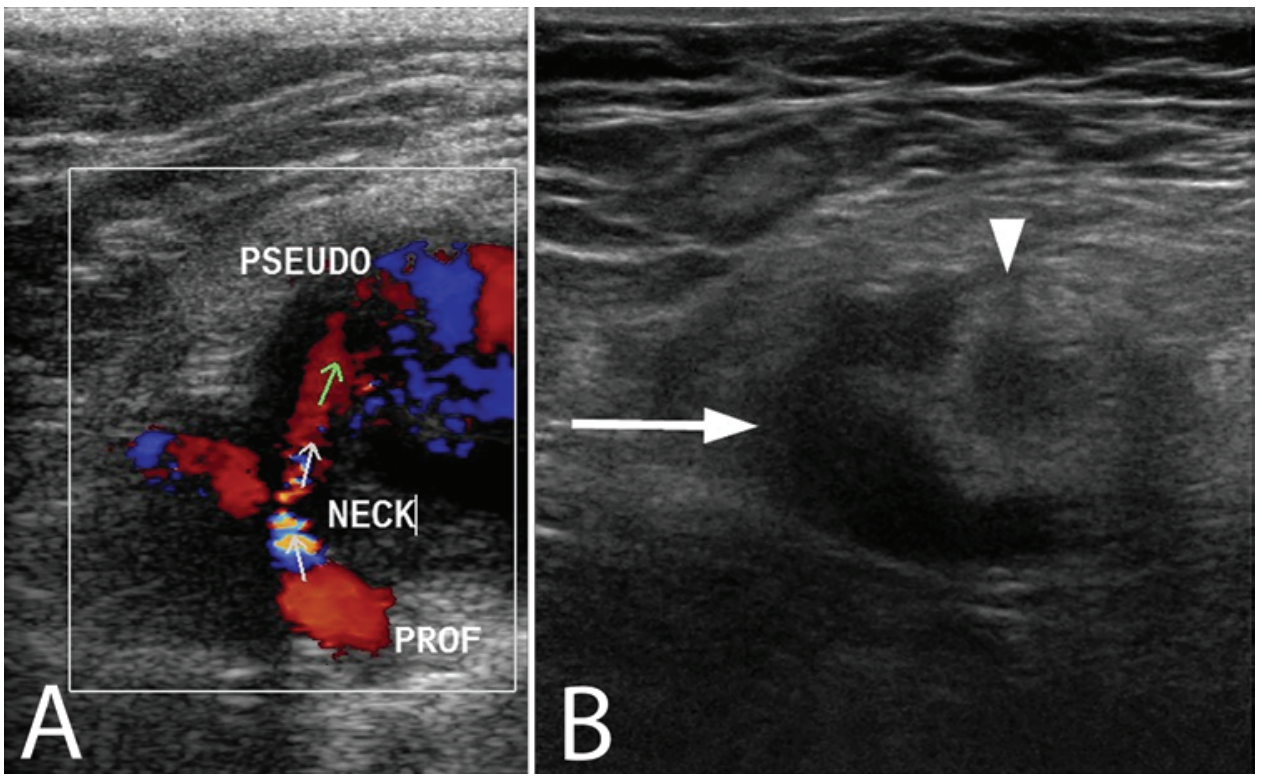

FIG. 5. A: Duplex ultrasound image shows a large pseudoaneurysm (pseudo) arising from the left profunda (prof) femoral artery with a clearly demonstrated neck and to-and-fro Doppler signal measuring approximately $6 \mathrm{~mm}$ in diameter. B: The pseudoaneurysm (arrow) dissects and displaces into the adjacent femoral nerve (arrowhead).

most importantly, in the diagnosis of axonotmesis/neurotmesis when neurophysiological evaluation could not.

In Case 1, we were able to accurately diagnose both tibial and, previously unrecognized, peroneal nerve transections with end-bulb neuromas using HRU in a patient who suffered a devastating leg injury. In addition, HRU showed the precise level of injury at the bifurcation of the sciatic nerve. The anatomical detail and extent of injury shown on HRU images matched the findings from MRI, but HRU showed the anatomy of individual fascicles and demonstrated a clear border between normal and abnormal tissue when MRI could not (Fig. 2). In a prospective study of 22 patients with lower-extremity nerve injuries,

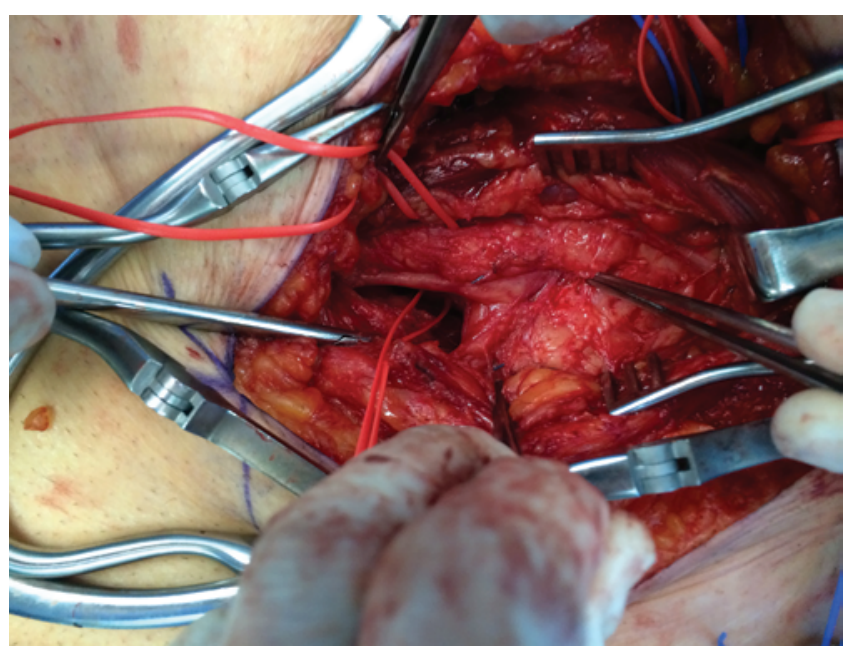

FIG. 6. Intraoperative image exposing the left femoral nerve and associated vasculature, highlighted with red rubber ties. The incision extends into the coronal plane. The femoral and profunda femoral arteries lie medial to the nerve.
Cokluk and Aydin ${ }^{10}$ concluded that visualization of lowerextremity peripheral nerves using ultrasound was good or excellent in $95 \%$ of the cases..$^{10}$ In addition, these authors were able to visualize and diagnosis stump neuromas in nearly $80 \%$ of the cases with only a $5-\mathrm{MHz}$ probe..$^{10}$

Case 2 showed iatrogenic pseudoaneurysm formation in a patient after a cardiac catheterization procedure, which displaced and dissected into the left femoral nerve. Although MRI accurately showed the femoral nerve involvement and the adjacent signal abnormality from the pseudoaneurysm, the femoral profunda pseudoaneurysm was shown more clearly on ultrasound using color Doppler imaging, and the displacement and dissection of the femoral nerve were clearly displayed. Although there are case reports in the literature describing peripheral nerve injury caused by pseudoaneurysms, there have been no large-scale studies evaluating the ability of ultrasound to identify and assess these lesions accurately.,17 We believe that neuronal damage secondary to vascular malformations can be well assessed with HRU, and further study is warranted.

Case 3 describes an ulnar nerve lesion for which ultrasound was used not only to confirm failure of the original graft but also to follow-up the integrity of the subsequent sural nerve-grafting procedure (Fig. 7C). Although ultrasound cannot detect peripheral nerve regeneration physiologically, the anatomical continuity of the nerve-grafting procedure can be confirmed. To our knowledge, no studies to date have critically evaluated the ability of ultrasound to assess graft integrity after surgical repair of a nerve gap. In general, ultrasound has been very effective in characterizing ulnar nerve pathology. In a prospective study of 91 patients with ulnar neuropathy, Filippou et al. ${ }^{11}$ showed HRU to successfully reveal detailed anatomy of the ulnar nerve. They also demonstrated the ability of HRU to distinguish several etiologies of ulnar neuropa- 

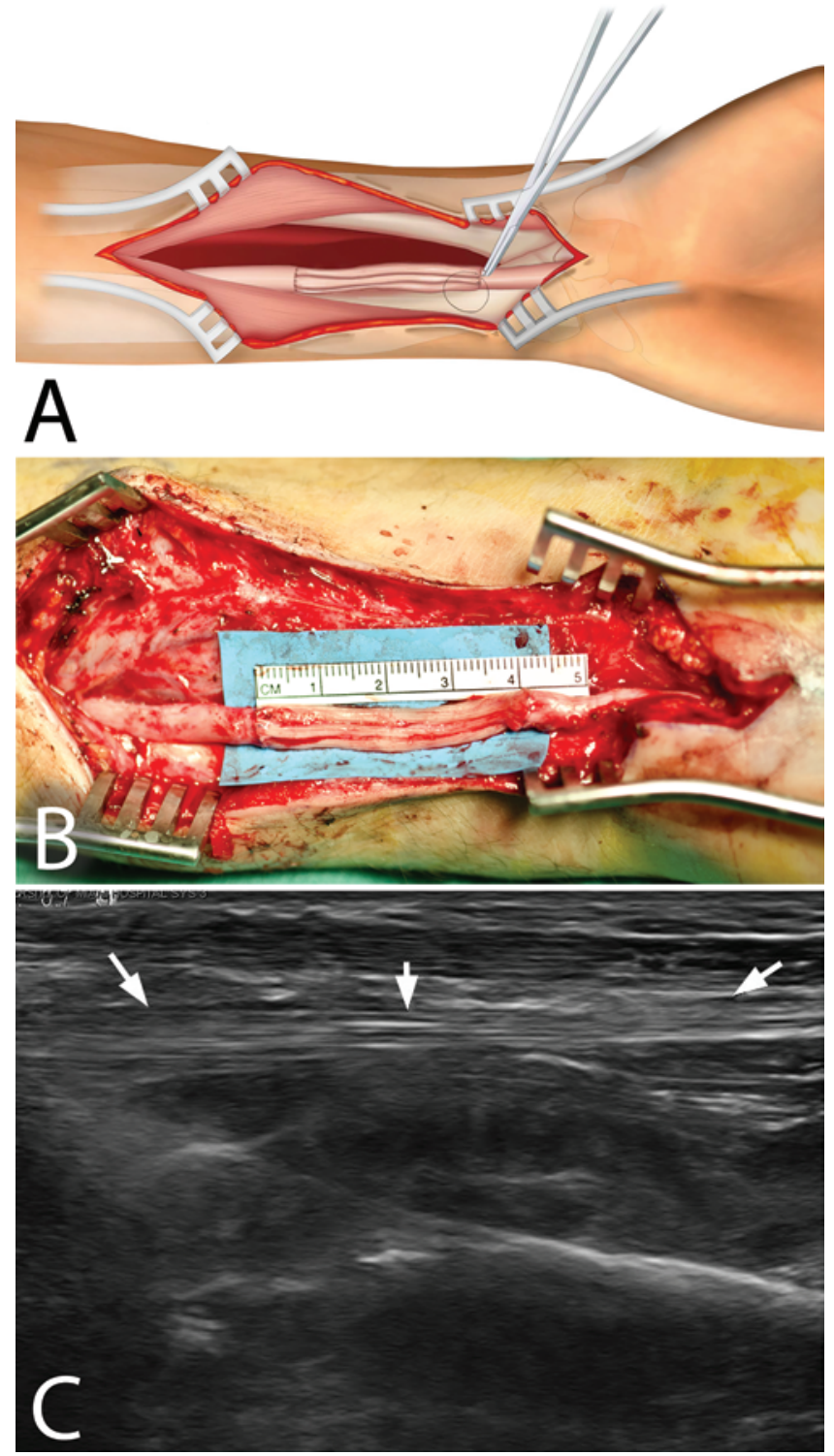

FIG. 7. A: Graphic illustration of intraoperative repair demonstrating cable grafts sutured into the nerve defect in continuity. Copyright University of Miami Miller School of Medicine. Published with permission. B: Intraoperative view after ulnar nerve repair with sural nerve allografts. From Berrocal et al: Limitations of nerve repair of segmental defects using acellular conduits. J Neurosurg 119:733-738, 2013. Published with permission. C: Sagittal ultrasound image of the left ulnar nerve repaired with sural nerve grafts 12 months after repair showing fascicular profiles and the absence of a neuroma.

thy such as subluxation and the presence of osteophytes and osseous lesions, articular ganglia, and posttraumatic lesions. ${ }^{11}$ A separate study by $\mathrm{Ng}$ et al. ${ }^{19}$ found that HRU is of greater use than nerve-conduction studies in the localization of ulnar neuropathy both at the elbow and outside the elbow in cases of traumatic ulnar neuropathy. Thus, HRU can accurately evaluate the relationship of the ulnar nerve with the adjacent soft tissues and osseous structures by using dynamic maneuvers without sacrificing excellent definition of the nerve and surrounding structures. ${ }^{11}$ In our case, the ultrasound images clearly showed contigu-
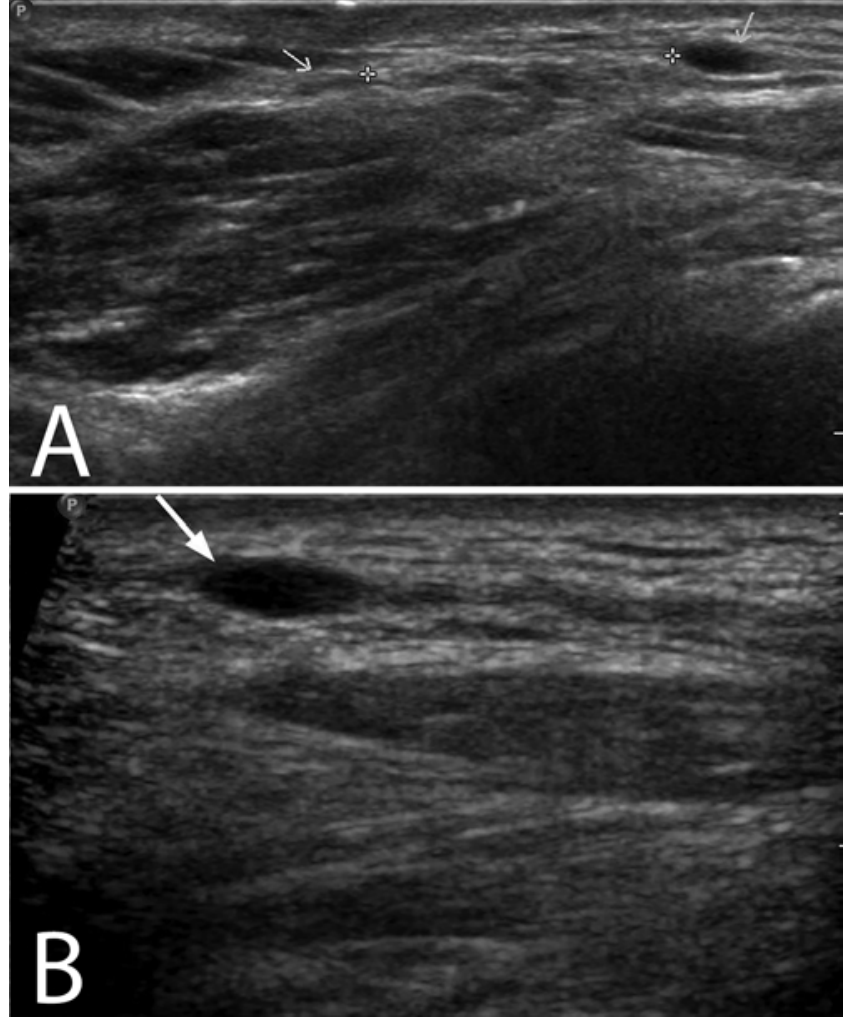

FIG. 8. A: Sunderland Grade 5 injury of the spinal accessory nerve in the lateral aspect of the posterior triangle of the neck, with a gap (calipers) measuring approximately $1.5 \mathrm{~cm}$ between the severed nerve ends (white arrows), just medial to the edge of the trapezius muscle. B: At the proximal nerve stump, there is a 4-mm end-bulb neuroma (white arrow).

ous and well-positioned sural grafts after nerve-gap repair (Fig. 7C). We believe that HRU would be a valuable tool in the postoperative stage, and this is an interesting topic for further research.

Case 4 describes the case of a woman who suffered a Sunderland Grade 5 injury to her spinal accessory nerve after surgical excision of a schwannoma. HRU clearly showed the accessory nerve transection and an end-bulb neuroma (Fig. 8). Bodner et al. ${ }^{6}$ described 4 cases of patients with accessory nerve palsy diagnosed directly or indirectly by trapezius muscle atrophy. Accessory nerve palsy causes dysfunction, weakness, and pain of the muscle, and the patient may present with a dropping shoulder or winged scapula on examination. ${ }^{6}$ The most common cause of accessory nerve injury is iatrogenic, for example with tumor resection or biopsy (as seen in our case), lymph node biopsy, or carotid endarterectomy. ${ }^{13,25} \mathrm{~A}$ review of the literature revealed that the use of HRU in diagnosing accessory nerve injuries has not been well studied.

Ordering physicians should be aware of the limitations inherent to the sonographic assessment of peripheral nerves. Ultrasound is operator dependent and requires knowledge of anatomy and subtle sonographic findings that involve structures that are only a few millimeters in size. With a linear-array transducer of $<7 \mathrm{MHz}$, the resolution is inherently limited in deeper tissues, particularly when the nerve lies deeper than $3 \mathrm{~cm} .{ }^{24}$ This limitation 
can be overcome in areas such as the supraclavicular brachial plexus, as demonstrated by Gruber et al., ${ }^{12}$ by using more powerful broadband linear-array transducers. Subtle abnormalities in deeper nerves may be harder to assess than with MRI, in which there is only signal abnormality but no change in nerve caliber. Finally, in our experience, changes in the surrounding muscle, such as subtle denervation edema or fatty atrophy, may be more difficult to appreciate with ultrasound than with MRI.

Ultrasound is becoming an increasingly important part of the diagnostic evaluation of peripheral nerve injuries despite the aforementioned limitations, and there are several well-described examples in the literature indicating that ultrasound may be the preferred imaging modality for assessing peripheral nerve lesions. High-frequency ultrasound can detect tiny abnormalities that simply cannot be shown by standard clinical MRI techniques, because the axial resolution is much better than is currently achievable with clinical MRI scanners. The axial resolution of a $10-\mathrm{MHz}$ probe is approximately $150 \mu \mathrm{m}$, much better than is currently achievable with clinical MRI scanners. ${ }^{18}$ In our experience, ultrasound is better able to delineate the tiny fascicles in the ulnar (Fig. 7C) spinal accessory nerves (Fig. 8A), which are difficult to discern on MRI. MRI is relatively contraindicated in patients with cardiac pacemakers and certain metal implants, and many patients find long MRI examinations uncomfortable because of claustrophobia or pain caused by immobilization. ${ }^{2}$ There are no contraindications for ultrasound examination. HRU also offers the benefit of dynamic imaging, which can show real-time changes, such as ulnar nerve subluxation during elbow flexion. ${ }^{14}$ Ultrasound also offers a more flexible field of view, an advantage over MRI when evaluating structures that have a long course in the body, such as peripheral nerves. ${ }^{18}$ Sonography also may be preferred over MRI when there is surgical hardware adjacent to the site of nerve injury, because it is not hampered by metallic

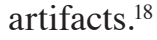

\section{Conclusions}

We present here 4 cases in which ultrasound guided the surgical management of patients in our practice. Additional research in the use of HRU in the evaluation of peripheral nerve injuries is warranted. Specifically, more data on the efficacy of HRU in aiding in the diagnosis of injuries to the spinal accessory nerve and peripheral nerve injuries caused by iatrogenic and traumatic pseudoaneurysms are needed. High-resolution ultrasound has been shown to be a useful tool in the diagnosis of peripheral nerve injuries when used in combination with physical examination and EMG. Ultrasound also provides dynamic imaging, is costefficient, and is more widely available than MRI.

\section{Acknowledgment}

We acknowledge Roberto Constantino Suazo for his assistance with figure submission.

\section{References}

1. Alaqeel A, Alshomer F: High resolution ultrasound in the evaluation and management of traumatic peripheral nerve injuries: review of the literature. Oman Med J 29:314-319, 2014

2. Bangard C, Paszek J, Berg F, Eyl G, Kessler J, Lackner K, et al: MR imaging of claustrophobic patients in an open 1.0T scanner: motion artifacts and patient acceptability compared with closed bore magnets. Eur J Radiol 64:152-157, 2007

3. Beekman R, Visser LH: High-resolution sonography of the peripheral nervous system - a review of the literature. Eur J Neurol 11:305-314, 2004

4. Berrocal YA, Almeida VW, Levi AD: Limitations of nerve repair of segmental defects using acellular conduits. J Neurosurg 119:733-738, 2013

5. Bianchi S: Ultrasound of the peripheral nerves. Joint Bone Spine 75:643-649, 2008

6. Bodner G, Harpf C, Gardetto A, Kovacs P, Gruber H, Peer $\mathrm{S}$, et al: Ultrasonography of the accessory nerve: normal and pathologic findings in cadavers and patients with iatrogenic accessory nerve palsy. J Ultrasound Med 21:1159-1163, 2002

7. Chen L, Peng F, Wang T, Chen D, Yang J: Traumatic pseudoaneurysm of axillary artery combined with brachial plexus injury. PLoS One 9:e113099, 2014

8. Chhabra A, Carrino J: Current MR neurography techniques and whole-body MR neurography. Semin Musculoskelet Radiol 19:79-85, 2015

9. Chiou HJ, Chou YH, Chiou SY, Liu JB, Chang CY: Peripheral nerve lesions: role of high-resolution US. Radiographics 23:e15, 2003

10. Cokluk C, Aydin K: Ultrasound examination in the surgical treatment of lower extremity peripheral nerve injuries: part II. Turk Neurosurg 17:197-201, 2007

11. Filippou G, Mondelli M, Greco G, Bertoldi I, Frediani B, Galeazzi M, et al: Ulnar neuropathy at the elbow: how frequent is the idiopathic form? An ultrasonographic study in a cohort of patients. Clin Exp Rheumatol 28:63-67, 2010

12. Gruber H, Glodny B, Galiano K, Kamelger F, Bodner G, Hussl H, et al: High-resolution ultrasound of the supraclavicular brachial plexus-can it improve therapeutic decisions in patients with plexus trauma? Eur Radiol 17:1611-1620, 2007

13. Harpf C, Rhomberg M, Rumer A, Rainer C, Hussl H: [Iatrogenic lesion of the accessory nerve in cervical lymph node biopsy.] Chirurg 70:690-693, 1999 (Ger)

14. Khoury V, Cardinal E, Bureau NJ: Musculoskeletal sonography: a dynamic tool for usual and unusual disorders. AJR Am J Roentgenol 188:W63-W73, 2007

15. Kuntz C IV, Blake L, Britz G, Filler A, Hayes CE, Goodkin $R$, et al: Magnetic resonance neurography of peripheral nerve lesions in the lower extremity. Neurosurgery 39:750-757, 1996

16. Lee FC, Singh H, Nazarian LN, Ratliff JK: High-resolution ultrasonography in the diagnosis and intraoperative management of peripheral nerve lesions. J Neurosurg 114:206-211, 2011

17. Lijftogt N, Cancrinus E, Hoogervorst ELJ, Mortel RH, Vries JP: Median nerve neuropraxia by a large false brachial artery aneurysm. Vascular 22:378-380, 2014

18. Nazarian LN: The top 10 reasons musculoskeletal sonography is an important complementary or alternative technique to MRI. AJR Am J Roentgenol 190:1621-1626, 2008

19. Ng ES, Vijayan J, Therimadasamy AK, Tan TC, Chan YC, Lim A, et al: High resolution ultrasonography in the diagnosis of ulnar nerve lesions with particular reference to posttraumatic lesions and sites outside the elbow. Clin Neurophysiol 122:188-193, 2011

20. Noble J, Munro CA, Prasad VS, Midha R: Analysis of upper and lower extremity peripheral nerve injuries in a population of patients with multiple injuries. J Trauma 45:116-122, 1998

21. Padua L, Di Pasquale A, Liotta G, Granata G, Pazzaglia C, 
Erra C, et al: Ultrasound as a useful tool in the diagnosis and management of traumatic nerve lesions. Clin Neurophysiol 124:1237-1243, 2013

22. Peer S, Bodner G, Meirer R, Willeit J, Piza-Katzer H: Examination of postoperative peripheral nerve lesions with highresolution sonography. AJR Am J Roentgenol 177:415-419, 2001

23. Taylor CA, Braza D, Rice JB, Dillingham T: The incidence of peripheral nerve injury in extremity trauma. Am J Phys Med Rehabil 87:381-385, 2008

24. Toros T, Karabay N, Ozaksar K, Sugun TS, Kayalar M, Bal E: Evaluation of peripheral nerves of the upper limb with ultrasonography: a comparison of ultrasonographic examination and the intra-operative findings. J Bone Joint Surg Br 91:762-765, 2009

25. Tucker JA, Gee W, Nicholas GG, McDonald KM, Goodreau JJ: Accessory nerve injury during carotid endarterectomy. J Vasc Surg 5:440-444, 1987

26. Zhu J, Liu F, Li D, Shao J, Hu B: Preliminary study of the types of traumatic peripheral nerve injuries by ultrasound. Eur Radiol 21:1097-1101, 2011

\section{Disclosure}

Dr. Levi reports that he has received teaching honoraria from Medtronic and DePuy Synthes.

\section{Author Contributions}

Conception and design: all authors. Acquisition of data: all authors. Analysis and interpretation of data: all authors. Drafting the article: all authors. Critically revising the article: all authors. Reviewed submitted version of manuscript: Levi, Zeidenberg, Jose, Subhawong. Approved the final version of the manuscript on behalf of all authors: Levi. Study supervision: Levi.

\section{Correspondence}

Allan D. Levi, Department of Neurological Surgery, University of Miami Miller School of Medicine, Lois Pope Life Center, 1095 NW 14th Terrace (D4-6), Miami, FL 33136. email: alevi@ med.miami.edu. 